\title{
Foetal Basal Forebrain Cell Type Transplants and Behavioural Recovery
}

\author{
E.J. Bradbury, T.R. Kershaw, R.M. Marchbanks and J.D. Sinden \\ Departments of Neuroscience and Psychology, Institute of Psychiatry, \\ De Crespigny Park, Denmark Hill, London SE5 8AF, UK
}

Cholinergic rich foetal basal forebrain transplants in the rat brain are known to have a beneficial effect in restoring cognitive function after excitotoxic damage to the forebrain cholinergic projection system. The heterogeneous nature of a cholinergic rich graft, however, makes it difficult to interpret behavioural effects solely in terms of cholinergic function. Recent work has attempted to characterize the protein composition of behaviourally effective and ineffective transplants. A glial protein (GFAP) was found to be enhanced in rats with basal forebrain grafts and levels of GFAP were positively correlated with behavioural recovery (Wets et al., J Neurochem, 1991). In this experiment we compared the behavioural effectiveness of grafts of primary post mitotic glial cultures with primary neuronal cultures, both prepared from foetal basal forebrain, transplanted bilaterally into neocortex and hippocampus. Transplants of whole foetal basal forebrain and sham transplants were made as positive and negative controls, respectively. Before transplantation animals had received lesions of the ascending cortical cholinergic projections using the excitotoxin AMPA. Performance on a T-maze win-shift spatial memory task showed no initial lesion effect. Introducing a 30 second delay produced a small, but not significant, difference between lesioned and control animals. After transplantation marginal differences were apparent between groups but further training resulted in approximately equal performances. However, following a four month period of no training, to allow for full transplant growth, the task was changed to a reversal, winstay paradigm and differences between groups became highly significant. Animals with trans- plants of glial cells performed as well as those with whole foetal basal forebrain transplants, suggesting that glial cells are an important factor in the amelioration of cognitive deficits by basal forebrain transplants. Both these groups reached the same level of performance as control animals. Performance of animals with primary neuronal transplants, however, was even poorer than the sham transplanted animals. Choline acetyltransferase (ChAT) assays indicated that the AMPA lesion had successfully damaged the cholinergic system, as measured by a depletion of activity in cortex and hippocampus in the lesion alone (sham) group. In regions containing whole basal forebrain and neuronal cell transplants ChAT activity had increased to a significantly higher level than the sham group. In regions containing glial cell transplants ChAT activity was not significantly different from the sham group levels which suggests that something other than cholinergic innervation was important for this group's behavioural recovery. GFAP amounts were determined in brain regions at the transplantation sites using the ELISA technique. Differences between groups were not significant but a trend for higher levels of GFAP in the three transplant groups was apparent as compared to the control and sham groups, with the highest amounts found in the glial transplant group. A neuronal marker (NSE) was assayed by immunoblotting and was present in equal amounts in all groups. These biochemical observations of the transplant sites, together with the behavioural findings, have highlighted the importance of glial cells for behavioural recovery following excitotoxic damage to the cholinergic system. Further, the restoration of 
cognitive function does not appear to be dependent upon a restoration of cholinergic activity. See Figure for group comparisons, expressed as a percentage of controls.

$B F=$ Basal forebrain; $A S T R=A$ strocyte; NEUR=Neuronal; SHAM=Sham transplant; HIPP=Hippocampus; $F C=$ Frontal cortex; ChAT=Choline acetyl transferase; GFAP $=$ Glial fibrillary acidic protein

A summary of behavioural and biochemical data showing a comparison of groups, expressed as a percentage of the control group. The baseline (100\%) represents the control group. E.g. the ASTR group, in both HIPP and FC, is almost level with controls on behavioural performance (93\%); has lower ChAT activity (75\% and $45 \%)$, and an increase in GFAP $(115 \%$ and $131 \%)$.
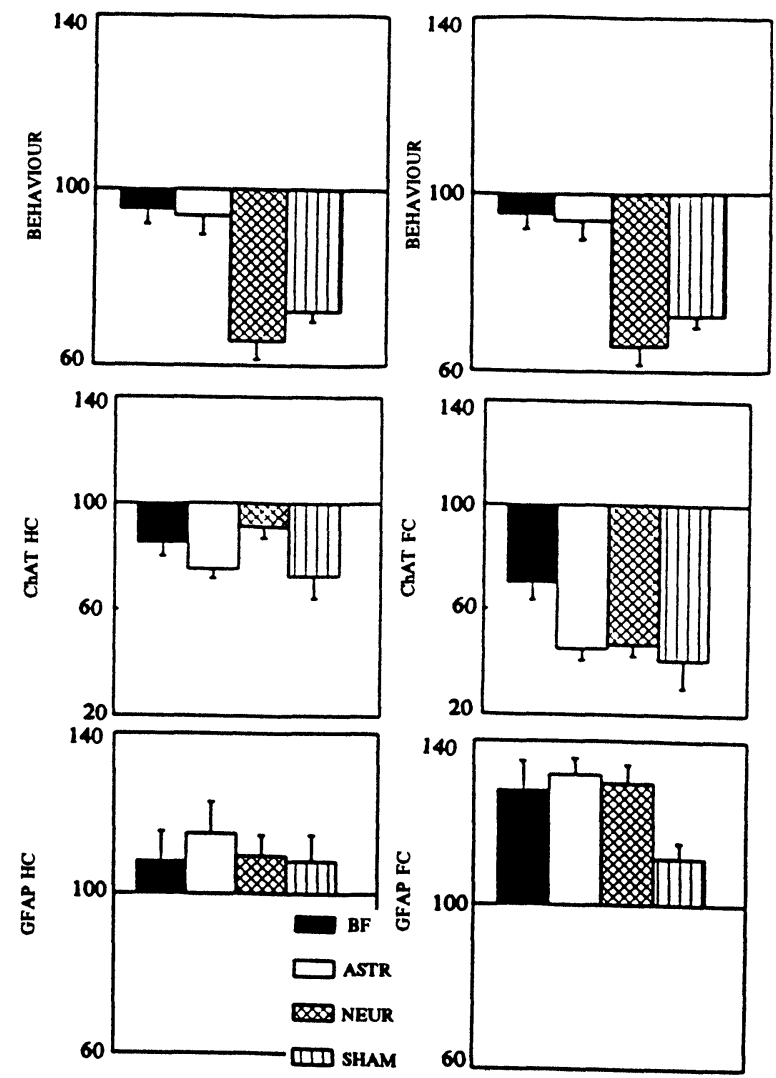

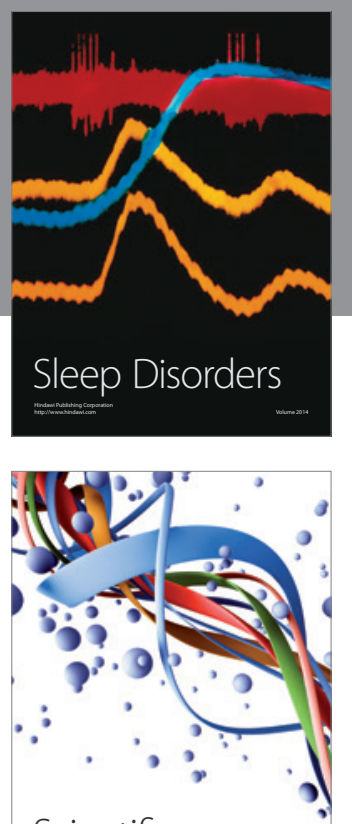

Scientifica
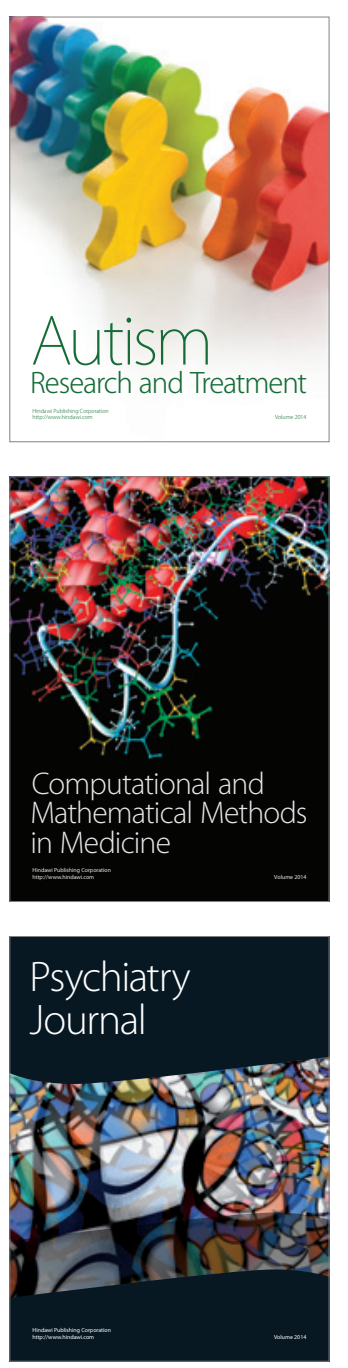
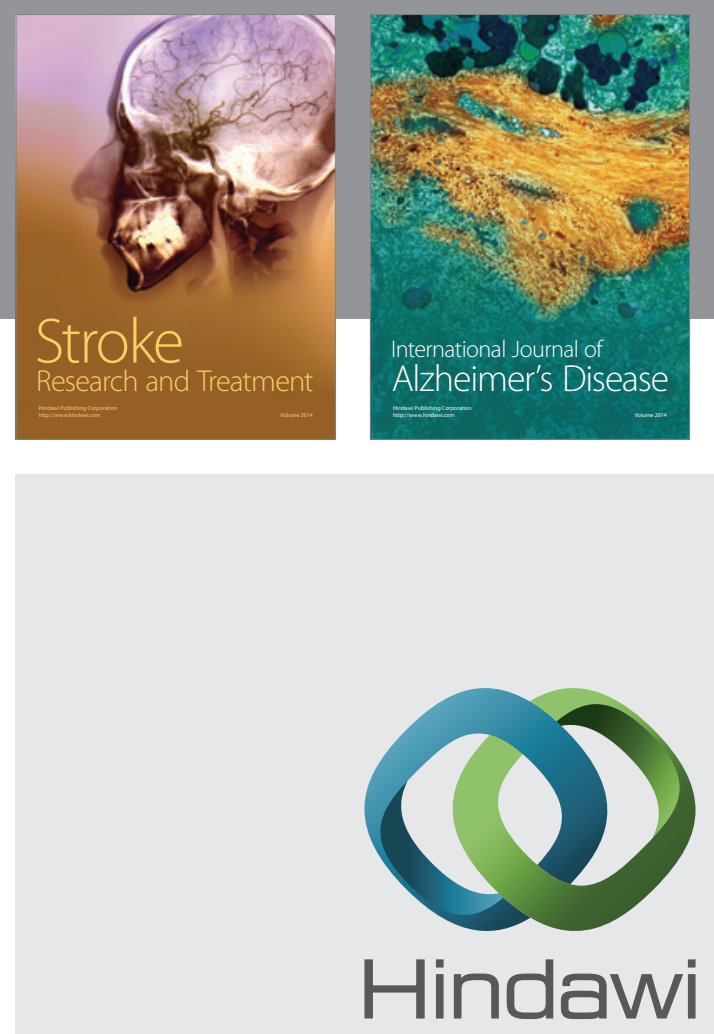

Submit your manuscripts at

http://www.hindawi.com
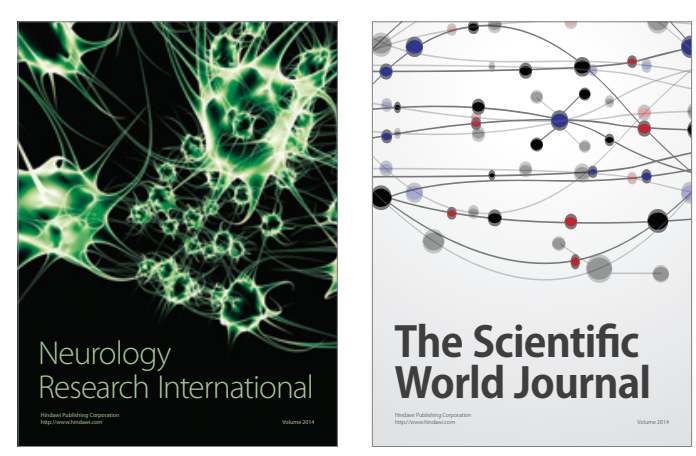

The Scientific World Journal

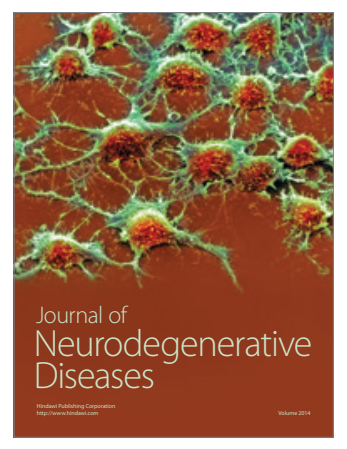

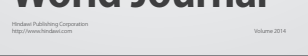

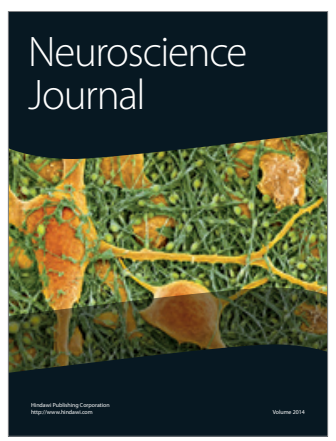

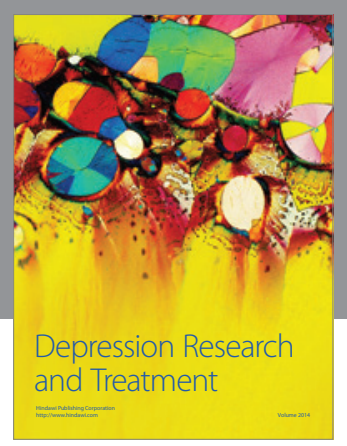
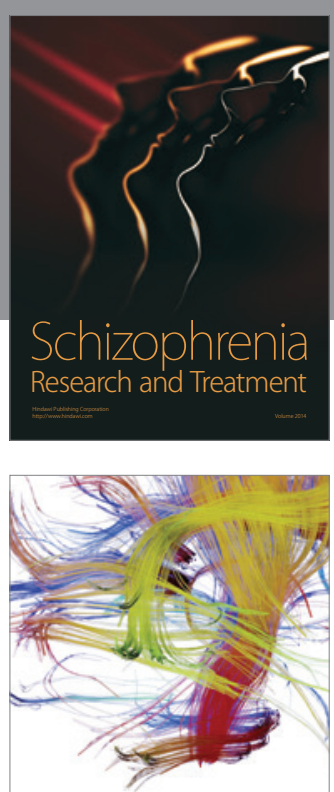

Brain Science

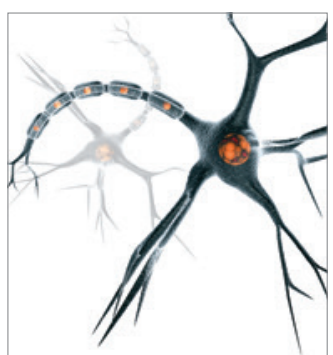

Neural Plasticity
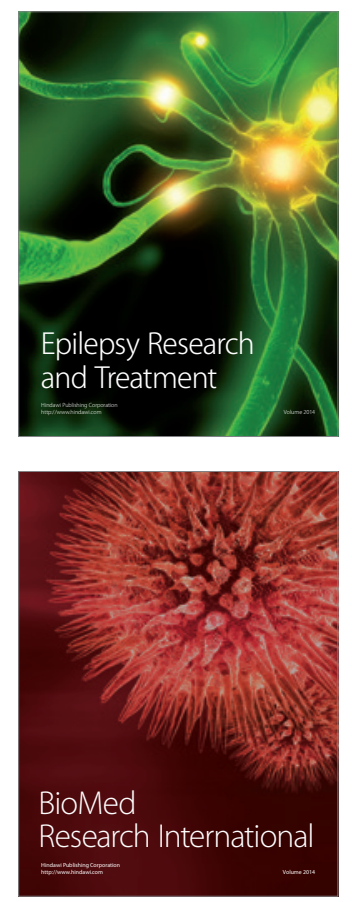

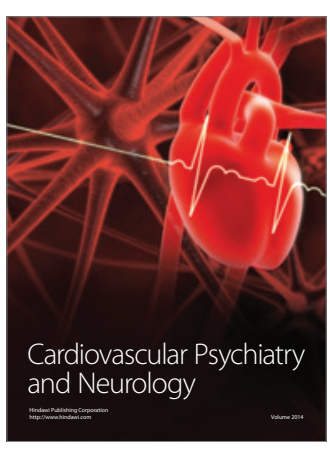

Parkinson's

Disease
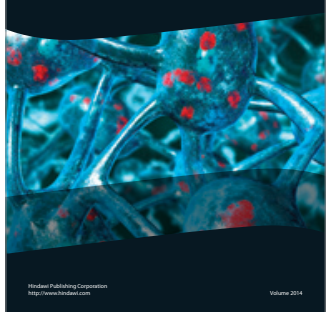